\title{
Thymidine phosphorylase exerts complex effects on bone resorption and formation in myeloma
}

\author{
Huan Liu ${ }^{1,}$, Zhiqiang Liu ${ }^{1},{ }^{*}$, Juan $\mathrm{Du}^{2}, \mathrm{Jin} \mathrm{He}^{1}$, Pei Lin ${ }^{3}$, Behrang Amini ${ }^{4}$, Michael W. \\ Starbuck $^{5}$, Nora Novane ${ }^{5}$, Jatin J. Shah ${ }^{1}$, Richard E. Davis ${ }^{1}$, Jian Hou ${ }^{2}$, Robert F. Gagel ${ }^{6}$, \\ and Jing Yang ${ }^{1, \dagger}$ \\ ${ }^{1}$ Department of Lymphoma and Myeloma, Division of Cancer Medicine, Center for Cancer \\ Immunology Research, The University of Texas MD Anderson Cancer Center, Houston, Texas \\ 77030, USA \\ 2Department of Hematology, The Myeloma \& Lymphoma Center, Changzheng Hospital, The \\ Second Military Medical University, Shanghai, China \\ ${ }^{3}$ Department of Hematopathology, Division of Pathology and Laboratory Medicine, The University \\ of Texas MD Anderson Cancer Center, Houston, Texas 77030, USA \\ ${ }^{4}$ Department of Diagnostic Radiology, Diagnostic Imaging, The University of Texas MD Anderson \\ Cancer Center, Houston, Texas 77030, USA \\ ${ }^{5}$ Department of Genitourinary Medical Oncology, Division of Cancer Medicine, The University of \\ Texas MD Anderson Cancer Center, Houston, Texas 77030, USA \\ ${ }^{6}$ Department of Endocrine Neoplasia and Hormonal Disorders, Division of Internal Medicine, The \\ University of Texas MD Anderson Cancer Center, Houston, Texas 77030, USA
}

\begin{abstract}
Myelomatous bone disease is characterized by the development of lytic bone lesions and a concomitant reduction in bone formation, leading to chronic bone pain and fractures. To understand the underlying mechanism, we investigated the contribution of myeloma-expressed thymidine phosphorylase (TP) to bone lesions. In osteoblast progenitors, TP upregulated the methylation of $R U N X 2$ and osterix, leading to decreased bone formation. In osteoclast progenitors, TP upregulated the methylation of IRF8, thereby enhanced expression of NFATc1, leading to increased bone resorption. TP reversibly catalyzes thymidine into thymine and 2DDR. Myeloma-secreted 2DDR bound to integrin $\alpha_{V} \beta_{3} / a_{5} \beta_{1}$ in the progenitors, activated PI3K/Akt signaling, and increased DNMT3A expression, resulting in hypermethylation of $R U N X 2$, osterix,
\end{abstract}

\footnotetext{
${ }_{*}^{\dagger}$ Correspondence: jiyang @ mdanderson.org.

* These authors contributed equally to this work.

Author contributions: H.L. and J.Y. designed all experiments and wrote the manuscript; H.L., Z.L., J.D., and J. He performed all experiments and statistical analyses; P.L. and J.J.S. provided the myeloma patients' samples; R.E.D., R.F.G., J. Hou, and B.A. provided critical suggestions; and M.W.S. and N.N. provided the injection technology and osteo-data analysis. All authors reviewed the final manuscript.

Competing interests: The authors have no competing financial interests.

Data and materials availability: Myeloma cell lines ARP-1, CAG, and ARK were kindly provided by University of Arkansas For Medical Sciences. Modified cell lines can be obtained from the corresponding authors through a material transfer agreement.
} 
and IRF8. This study elucidates an important mechanism for myeloma-induced bone lesions, suggesting that targeting TP may be a viable approach to healing resorbed bone in patients. As TP overexpression is common in bone-metastatic tumors, our findings could have additional mechanistic implications.

\section{INTRODUCTION}

Bone is constantly being remodeled in a process where osteoclasts resorb bone, then osteoblasts deposit type I collagen and other proteins in the resorbed lacunae, and lastly the collagen mineralizes to form bone $(1,2)$. The first partner in this pas de deux is the osteoclast, which arises from hematopoietic monocytic precursors and resorbs bone. The formation of osteoclasts requires the cytokine receptor activator of nuclear factor- $\mathrm{\kappa B}$ ligand (RANKL) and macrophage colony-stimulating factor (M-CSF) (3). RANKL enhances the expression of nuclear factor of activated T-cells, cytoplasmic 1 protein (NFATc1), a transcriptional factor that upregulates the expression of osteoclast differentiation-associated genes, such as tartrate-resistant acid phosphatase (TRAP), calcitonin receptor (CALCR), and cathepsin $\mathrm{K}(C T S K)$; whereas the transcriptional factor interferon regulatory factor 8 (IRF8) can suppress RANKL-induced NFATc1 expression (4). The second player in the remodeling cycle is the osteoblast, which is differentiated from mesenchymal stem cells (MSC). This process requires the activation of core-binding factor a-1/runt-related transcription factor 2 (RUNX2) and osterix, which stimulate the expression of osteoblast differentiation-associated genes, such as bone gamma-carboxyglutamic acid-containing protein $(B G L A P)$, alkaline phosphatase $(A L P)$, and collagen type I a 1 (COL1AI).

This delicate balance between osteoclast-mediated bone resorption and osteoblast deposition of matrix is disrupted in certain types of malignancies, including multiple myeloma and solid tumors, such as breast and lung cancer, among others $(5,6)$. In multiple myeloma, tumor cells secrete RANKL or stimulate the release of RANKL by surrounding stromal cells, leading to enhanced osteoclast differentiation. Myeloma cells can also secrete dickkopf-related protein 1 (DKK1), which inhibits the Wnt/ $\beta$-catenin signaling pathway and suppresses osteoblast differentiation. Attempts to target RANKL and DKK1 in myeloma therapeutically have achieved only modest success. For an example, the anti-resorptive agent denosumab (a monoclonal antibody against RANKL) was examined in a Phase III trial, but only shown a moderately affected myeloma-induced lytic lesions (7). BHQ880 (a monoclonal antibody against DKK-1) is now in a Phase I/II study, but its application in myeloma fails to restore new bone formation (8). Thus bisphosphonates, which suppress osteoclast function, remain the mainstay in treatment of myeloma-induced bone disease. Unfortunately, bisphosphonates are less than fully effective and cause osteonecrosis of the jaw in $2-5 \%$ of treated patients (7). The goal of the current studies is to identify other factors produced by myeloma cells, which regulate both resorption and formation, and to determine whether this information can be used to prevent myeloma-induced bone disease.

Thymidine phosphorylase (TP), also called platelet-derived endothelial cell growth factor, is an enzyme that can reversibly catalyze the conversion of thymidine to thymine and 2-deoxyD-ribose-1-phosphate (2DDR1P), which is further dephosphorylated into a smaller, more 
stable molecule, 2-deoxy-D-ribose (2DDR) (9). TP has been found in a wide range of normal tissues (10), and participates in wound healing and a variety of chronic inflammatory diseases (11). TP is highly expressed in many types of cancers, including lung and breast, and plays an important role in angiogenesis and anti-apoptosis $(12,13)$. Clinically, elevated levels of TP are associated with cancer aggressiveness and poor prognosis (11), but TP has never been implicated in the regulation of bone resorption or formation. In previous studies, we demonstrated that p38 MAPK activity in myeloma cells induces osteolytic bone lesions $(14,15)$ and others demonstrated that this signaling regulates TP expression $(16,17)$. We therefore hypothesized that the $T P$ gene plays a role in the pathogenesis of cancer-induced bone destruction in myeloma.

Here, we report an association of cancer-expressed TP with osteolytic bone lesions, as well as the ability of TP to orchestrate osteoclast-mediated resorption and decreased bone formation. Specifically, TP downregulated the expression of IRF8 and thereby activated RANKL-induced NFATc1 expression, leading to an increase in osteoclastogenesis and bone resorption. We further observed that myeloma-expressed TP suppressed osteoblastogenesis and bone formation by downregulating the expression of RUNX2 and osterix in human MSCs. Our findings not only elucidate a mechanism of cancer-induced suppression of osteoblast differentiation and activation of osteoclast differentiation and activity, but also implicate a potential therapeutic approach for cancer patients with osteolytic bone lesions by targeting TP.

\section{RESULTS}

\section{Myeloma-expressed TP enhances lytic bone lesions}

We confirmed the presence of myeloma $\left(\mathrm{CD} 138^{+}\right)$cells in tissue array biopsies, and found that $\mathrm{TP}^{+}$cells were significantly greater in the bone marrow from 14 myeloma patients than 14 healthy donors (Fig. 1, A and B). TP was expressed in a majority of bone marrow aspirates of primary myeloma cells (4 of 6 patients) and in a majority of established human myeloma cell lines (4 of 6), but not in aspirates of plasma cells from normal subjects (Fig. 1C).

To determine whether TP expression correlated with the frequency of bone lesions in myeloma patients, we performed two experiments using samples from tissue banks. In the first, there was a strong positive correlation between $T P$ gene expression in myeloma cells and bone lesion numbers in 52 patients (Fig. 2A); in the second, there was a robust positive correlation between TP immunohistochemistry and bone lesion numbers (Fig. 2B). TP expression was higher in myeloma cells from patients with high bone lesion scores than those from patients with low lesion numbers (Fig. 2C).

We then isolated primary myeloma cells from the bone marrow aspirates of 10 newly diagnosed patients. Based on the TP expression in myeloma cells as detected by western blot, the aspirates were separated into high and low $\mathrm{TP}$ expression groups: $\mathrm{TP}_{\text {high }}$ and $\mathrm{TP}_{\text {low }}$ ( $n=5$ patients' bone marrow aspirates/group) (fig. S1A). To determine the functional role of myeloma-expressed TP in lytic bone lesions, we injected $\mathrm{TP}_{\text {high }}$ or $\mathrm{TP}_{\text {low }}$ cells into human bone chips that had been implanted into severe combined immunodeficient (SCID)-hu mice. 
Mice with no tumor cells (but with bone chips implanted) were controls. More lytic lesions and a lower percentage of bone volume vs total volume (BV/TV) were observed in the bone chips of mice injected with $\mathrm{TP}_{\text {high }}$ than $\mathrm{TP}_{\text {low }}$ cells (Fig. 2, D and H). Moreover, injecting WT ARP-1 cells, which have high TP expression, into mouse femurs caused more lytic lesions than WT MM.1S cells, which have low TP expression (Fig. 2, E and H; fig. S1B). ARP-1 cells with TP knocked down by shRNAs (sh TP ARP-1) were injected into mouse femurs and caused fewer lytic lesions than ARP-1 cells with a non-targeted shRNA (shCtrl ARP-1) (Fig. 2, F and H; fig. S1C). Conversely, MM.1S cells expressing TP cDNA (TP MM.1S) caused more femur lesions than WT MM.1S cells expressing a control vector ( Vec MM.1S) (Fig. 2, G and H; fig. S1D). In sum, cells with high TP expression induce the formation of lytic lesions in mouse and human bone.

To determine whether lytic lesions result from a change in the tumor burden within the marrow milieu, we measured the levels of myeloma-secreted M-protein, a reflection of tumor burden, in the mouse sera. The modulation of TP expression in myeloma cells did not change the serum levels of M-protein (fig. S1E). In addition, there were no differences in viability or apoptosis in control and modified ARP-1 or MM.1S cell lines (fig. S1, F and G), indicating that changes in TP expression did not affect myeloma cell growth or survival. Together, these results reveal that myeloma cells express TP and enhance lytic bone lesions in patients and mice with myeloma.

\section{Myeloma-expressed TP enhances RANKL-induced osteoclastogenesis and bone resorption}

We studied the ability of myeloma-expressed TP to regulate osteoclast differentiation in vitro using a standard osteoclast differentiation protocol (18) in which $\mathrm{TRAP}^{+}$cell number and TRAP5b secretion were assessed in the presence of RANKL $(10 \mathrm{ng} / \mathrm{ml})$ and M-CSF (25 $\mathrm{ng} / \mathrm{ml}$ )—both of which are needed for osteoclast formation. Co-culture of preOCs with $\mathrm{TP}_{\text {high }}$ patient myeloma cells or WT ARP-1 cells induced higher numbers of multinuclear $\mathrm{TRAP}^{+}$cells (fig. S2A), more TRAP5b secretion (fig. S2B), and higher expression of the osteoclast genes TRAP, CALCR, and CTSK (fig. S2, C and D) than co-culture with cells expressing low levels of TP ( $\mathrm{TP}_{\text {low }}$ or WT MM.1S). Knockdown of TP expression in WT ARP-1 cells reduced osteoclast differentiation and activity (fig. S2, A, B, and E). In contrast, overexpression of TP in WT MM.1S cells enhanced the differentiation and activity (fig. S2, $\mathrm{A}, \mathrm{B}$, and F).

To assess the role of myeloma-expressed TP in osteoclast-mediated bone resorption in vivo, we stained myeloma-bearing human bone chips or mouse femurs for TRAP and analyzed the percentage of bone surface eroded by osteoclasts (ES/BS) and the percentage of bone surface covered with osteoclasts (Oc. S/BS). The percentage of ES/BS (Fig. 3A) and Oc. $\mathrm{S} / \mathrm{BS}$ were higher in the mice injected with myeloma cells that had high TP expression $\left(\mathrm{TP}_{\text {high }}\right.$, WT ARP-1, shCtrl ARP-1 or TPMM.1S) than those with low TP expression (TP ${ }_{\text {low }}$, sh TPARP-1, WT MM.1S or Vec MM.1S) (Fig. 3, A and B). These results indicate that myeloma-expressed TP enhances osteoclastogenesis.

In preOCs, myeloma cells have been shown to enhance the expression of NFATc1, a transcriptional factor that plays a pivotal role in osteoclast gene expression and can be 
upregulated by RANKL (19). We confirmed this effect of myeloma cells on NFATc1 expression in preOCs (fig. S3A), and further demonstrated that myeloma cells with high but not low TP downregulated expression of the transcription factor IRF8 in preOCs (fig. S3A). To determine whether IRF8 mediates TP-induced osteoclast differentiation, its expression in preOCs was knocked down using IRF8 shRNA (shIRF8). When co-cultured with WT ARP-1 cells, shIRF8-preOCs expressed higher levels of NFATc1 protein (fig. S3B) and secreted more TRAP5b (fig. S3C) than control preOCs. Thus, myeloma-expressed TP abrogated the inhibitory effect of $I R F 8$ on NFATc1 shown previously (3), leading to the promotion of osteoclast gene expression and osteoclastogenesis.

\section{Myeloma-expressed TP inhibits osteoblastogenesis in vitro and bone formation in vivo}

To determine whether myeloma-expressed TP regulates osteoblast differentiation in vitro, we co-cultured the precursors of osteoblasts, MSCs, with patient myeloma cells and myeloma cell lines in osteoblast medium for 2 weeks. MSCs cultured alone in this medium served as a positive control. Mature osteoblasts produced soluble alkaline phosphatase, were positive for Alizarin red S staining (which is indicative of osteoblast-mediated bone formation activity), and expressed osteoblast differentiation-associated genes (fig. S4). In line with previous studies (20), co-culture of MSCs with myeloma cells inhibited osteoblast activity, but co-culture with $\mathrm{TP}_{\text {low }}$ myeloma cells and low-TP-expressing myeloma cell lines (WT MM.1S, Vec MM.1S, or sh TPARP-1) had comparatively more mature osteoblasts than cells with high levels of TP (fig. S4).

To determine the role of myeloma-expressed TP in osteoblast-mediated bone formation in vivo, we collected myeloma-bearing human bone chips or mouse femurs and enumerated the osteoblasts localized on trabecular bone and counted the percentage of osteoid surface (OS/BS) and of bone surface lined with osteoblasts (Ob. S/BS). The percentages of OS/BS (Fig. 3C) and Ob. S/BS (Fig. 3D) were lower in the mice injected with myeloma cells

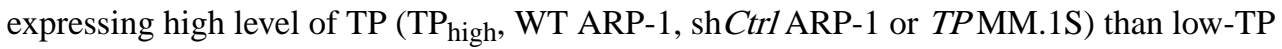
myeloma cells ( $\mathrm{TP}_{\text {low }}$, sh TP ARP-1, WT MM.1S or Vec MM.1S). In agreement with these data, bone formation rate was increased in mice injected with sh TP ARP-1 cells (Fig. 3E) and decreased in mice with TPMM.1S cells (Fig. 3F).

Transcription factors such as $R U N X 2$ and osterix promote osteoblast differentiation by upregulating the expression of osteoblast differentiation-associated genes (21). In line with previous studies (20), we observed that co-culture of MSCs with myeloma cells reduced the expression of RUNX2 and osterix (fig. S5). Moreover, the expression of these transcription factors was significantly lower when co-cultured with myeloma cells with high levels of TP than with lower TP levels (fig. S5A). Moreover, myeloma-expressed TP modulated the binding activity of RUNX2 and osterix to the promoter of osteoblast genes BGLAP and COL1A1 (fig. S5, B and C), a finding consistent with the effect of TP on bone formation.

\section{TP downregulates the expression of RUNX2, osterix, and IRF8 via hypermethylation of CpG islands}

DNA methylation of $\mathrm{CpG}$ dinucleotides is a key epigenetic modification that influences tissue- and context-specific gene expression (22) and is generally associated with gene 
silencing (23). To determine whether myeloma-expressed TP regulates the methylation of CpG islands (CGI) in RUNX2, osterix, and IRF8, we designed methylation-specific PCR and bisulfite sequencing PCR primers targeting their CpG-rich regions (Fig. 4A; table S2). Methylation of RUNX2 and osterix in MSCs and IRF8 in preOCs was higher in the coculture of myeloma cells with high TP expression (WT ARP-1, shCtrl ARP-1, and TPMM. 1S) than in the co-culture of those with low TP expression (sh TP ARP-1, WT MM.1S, and Vec MM.1S) (Fig. 4B). Bisulfite sequencing PCR analysis confirmed these results (Fig. 4, $\mathrm{C}-\mathrm{E}$ ), and similar methylation data were obtained from healthy individual and myeloma patient samples expressing different levels of TP (Fig. 4F).

DNA methyltransferases (DNMTs) are important for the methylation of gene promoters. We observed higher gene expression and enzyme activity of DNMT3A—but not DNMT1 or DNMT3B - in MSCs and preOCs co-cultured with high-TP myeloma cells (WT ARP-1, sh Ctrl ARP-1, and TPMM.1S) than with low-TP cells (sh TP ARP-1, WT MM.1S, and Vec MM.1S) (fig. S6, A and B). The clinical relevance was further studied by correlating $T P$ expression in myeloma cells from patients or number of bone lesions in these patients with $D N M T 3 A$ expression in MSCs and preOCs from the same patients. We found positive correlations between TPexpression and number of bone lesions with DNMT3A in MSCs or preOCs (fig. S6C). Collectively these data demonstrate that myeloma-expressed TP upregulates DNMT3A expression and activity in MSCs and preOCs, consequently enhancing methylation of CGIs in the promoters of RUNX2, osterix, and IRF8 genes, suppressing osteoblast differentiation, and increasing osteoclast activity. These signaling changes shift the balance of bone remodeling toward a net loss of bone tissue.

\section{TP upregulates DNMT3A expression through 2DDR-mediated signaling}

TP degrades thymidine into thymine and 2DDR1P; the unstable 2DDR1P is further dephosphorylated to 2DDR (11). Not unexpectedly, in vitro myeloma cells with high levels of TP secreted more 2DDR than low-TP cells (fig. S7A). Similarly, higher levels of 2DDR were observed in the serum of mice bearing high-TP myeloma cells (TPMM.1S, WT ARP-1) than in mice bearing low-TP cells (WT MM.1S, sh TP ARP-1) (fig. S7B).

To test whether 2DDR mediates TP-induced suppression of osteoblastogenesis, we cultured MSCs in osteoblast medium with incremental doses of 2DDR for 2 weeks. The addition of 2DDR downregulated ALP production, Alizarin red S staining, and the expression of osteoblast genes in a dose-dependent manner (fig. S7C). Furthermore, the addition of 2DDR decreased the expression of RUNX2 and osterix (Fig. 5A), and upregulated the expression and activity of DNMT3A in MSCs (Fig. 5B). Culture of MSCs in medium with 2DDR induced the hypermethylation of CGIs on the RUNX2 and osterix genes (Fig. 5C) and inhibited ALP production and Alizarin red S staining (Fig. 5D). Knockdown of DNMT3A in MSCs partially abrogated the effects of 2DDR (Fig. 5, C and D).

To examine the role of 2DDR in the activation of osteoclastogenesis, we cultured preOCs with RANKL (10 ng/ml) and M-CSF ( $25 \mathrm{ng} / \mathrm{ml})$ without or with escalating doses of 2DDR for 1 week. 2DDR enhanced multinuclear TRAP ${ }^{+}$cell numbers, TRAP5b secretion, and the expression of the osteoclast genes TRAP, CALCR, and CTSK in a dose-dependent manner (fig. S7D). 2DDR also reduced IRF8 expression (Fig. 5E), upregulated DNMT3A 
expression (Fig. 5F) and induced hypermethylation of CGIs in IRF8 (Fig. 5G). Knocking down DNMT3A in preOCs reversed 2DDR-mediated IRF8 hypermethylation (Fig. 5G) and increased osteoclast formation (Fig. 5H).

We next investigated the signaling pathways by which 2DDR may regulate DNMT3A expression. 2DDR is known to bind integrins $\alpha_{V} \beta_{3}$ and $\alpha_{5} \beta_{1}(11)$. We confirmed in the literature that all MSCs in this study express both integrins (24), and that preOCs express $a_{V} \beta_{3}$ (25). The addition of an antibody against either the $a_{5}$ subunit of $a_{5} \beta_{1}$ or the $a_{V}$ subunit of $\alpha_{V} \beta_{3}$, but not control IgG, to the co-culture of MSCs with 2DDR enhanced osteoblast formation; the addition of both anti- $a_{5}$ and anti- $a_{V}$ antibodies had a synergistic effect (fig. S8A). Moreover, application of both antibodies inhibited 2DDR-stimulated $D N M T 3 A$ expression in MSCs (fig. S8B), indicating that 2DDR regulates osteoblast differentiation and DNMT3A expression via $\alpha_{V} \beta_{3}$ and/or $\alpha_{5} \beta_{1}$.

Furthermore, we examined the ERK and PI3K/Akt signaling pathways, downstream of $a_{5} \beta_{1}$ and $\alpha_{V} \beta_{3}$. The addition of 2DDR to culture of MSCs upregulated the phosphorylated levels of Akt and the integrin-mediated downstream molecules focal adhesion kinase (FAK), Paxillin, and p130Cas, but did not change phosphorylated levels of ERK or nonphosphorylated levels of these molecules (fig. S8C). Akt phosphorylation induced by 2DDR was abrogated with antibodies against both $a_{5}$ and $a_{\mathrm{V}}$ (fig. S8D). An Akt inhibitor LY294002 blocked 2DDR-induced DNMT3A expression in MSCs (fig. S8E).

Addition of anti- $\mathrm{a}_{\mathrm{V}}$ antibodies to the culture of preOCs reduced 2DDR-induced TRAP5b secretion and DNMT3A expression (fig. S8, F-G). Addition of 2DDR upregulated the levels of phosphorylated Akt, FAK, Paxillin, and p130Cas in cultured preOCs (fig. S8H), and blocking $a_{V}$ with the antibodies (fig. S8I) reduced the phosphorylation of Akt. Similar to the results in MSCs, administration of LY294002 significantly reduced 2DDR-induced DNMT3A expression in preOCs (fig. S8J).

To validate the antibody blocking study, we knocked down $a_{V}$ and/or $a_{5}$ expression in MSCs using siRNAs (fig. S9A). Adding 2DDR to cultures of si $a V$ or sia 5 MSCs reduced osteoblast formation, DNMT3A expression, and Akt phosphorylation in MSCs (fig. S9, BD). The siRNAs against Akt1/2 blocked 2DDR-induced DNMT3A expression in MSCs (fig. S9E-F). Moreover, knockdown of $a_{\mathrm{V}}$ expression in preOCs reduced 2DDR-induced TRAP5b secretion and DNMT3A expression (fig. S9, G-I) and knockdown of Akt1/2 (fig. S9J) significantly reduced 2DDR-induced $D N M T 3 A$ mRNA (fig. S9, K-L). These results suggest that TP regulates 2DDR secretion from myeloma cells, and 2DDR enhances DNA methylation of $R U N X 2$ and osterix in MSCs and IRF8 in preOCs through the $a_{V} \beta_{3} / a_{5} \beta_{1^{-}}$ PI3K/Akt-DNMT3A signaling pathway.

\section{Inhibiting TP reduces myeloma-induced osteolytic bone lesions}

Toward a therapeutic, we asked whether inhibiting TP can prevent myeloma-induced osteolytic bone lesions. For this purpose, ARP-1 cells, which express high levels of TP, were directly injected into the femurs of SCID mice. Mouse serum was collected to measure circulating M-protein levels for monitoring tumor burden. When myeloma was established, mice were treated with vehicle control or TP inhibitors, 7-deazaxanthine (7DX) or tipiracil 
hydrochloride (TPI). ARP-1 cells caused osteolytic bone lesions and increased osteoclastogenesis, reduced bone volume and osteoblastogenesis (Fig. 6, A-D). TPI or 7DX treatment significantly reduced ARP-1 induced bone lesions (Fig. 6, A-D). Treatment with 7DX or TPI significantly reduced Dnmt3a expression in mouse MSCs or preOCs (Fig. 6E) and 2DDR levels in the serum of ARP-1 tumor-bearing mice (Fig. 6F).

Using two additional human myeloma cell lines, RPMI8226 (high TP expression) and U266 (low TP expression), we observed similar effects of TP inhibitors on myeloma-induced bone lesions both in vitro and in vivo (Fig. 7). Co-culture of RPMI8226 cells, secreting more 2DDR (Fig. 7A), increased osteoclast (Fig. 7B) and suppressed osteoblast (Fig. 7C) differentiation and activity, compared to those in co-culture of U266 cells (secreting less 2DDR). Treatment of RPMI8226-bearing mice with TPI or 7DX enhanced bone volume and osteoblastogenesis and reduced osteoclastogenesis (Fig. 7, D-F).

\section{DISCUSSION}

Our study reveals an important biological function of TP in the pathogenesis of myelomaassociated osteolytic bone lesions (Fig. 6G) and indicates that counteracting TP activity may be effective for prevention or treatment of osteolytic bone lesions in myeloma patients. We found that TP reversibly catalyzes conversion of thymidine into thymine and 2DDR. Myeloma-secreted 2DDR binds to integrins $a_{V} \beta_{3} / a_{5} \beta_{1}$ in osteoblast progenitors, activates PI3K/Akt signaling, and increases DNMT3A expression and methylation of $R U N X 2$ and osterix, leading to decreased obsteoblastogenesis. The secreted 2DDR also binds to integrin $a_{V} \beta_{3}$ in osteoclast progenitors, activates PI3K/Akt signaling, and increases DNMT3A expression and methylation of IRF\&, leading to increased NFATc1 expression and osteoclastogenesis. The net effect of TP is to suppress osteoblast-mediated bone formation and activate osteoclast-mediated bone resorption, the hallmarks of myeloma-induced bone disease.

DNA methylation regulation has been shown to be one of the most important regulatory mechanisms of gene expression (26). For instance, $\mathrm{CpG}$ methylation is catalyzed by a family of DNMTs (27), and DNMT3A and 3B specifically are able to modify un-methylated DNAs. However, the involvement of DNA methylation in osteoblast and osteoclast differentiation is unclear. Our results demonstrated that myeloma-expressed TP upregulated DNMT3A expression but not DNMT1 or 3B in MSCs via 2DDR, the metabolic product of thymidine, leading to highly methylated CGIs in RUNX2 and osterix gene promoters. Hypermethylation caused down-regulation of these two genes, resulting in decreased osteoblast-mediated bone formation — a hallmark of myeloma. In addition, myelomaexpressed TP enhanced osteoclast differentiation. TP/2DDR downregulated IRF8 expression in preOCs via DNA hypermethylation by DNMT3A, thereby enhancing RANKL-induced NFATc1 expression, demonstrating a critical role of DNA methylation in myeloma-induced activation of osteoclastogenesis. These results point to a new epigenetic mechanism underlying myeloma-induced bone disease.

Integrins are important for bone remodeling (28). For example, integrins $\alpha_{5} \beta_{1}$ and $a_{V} \beta_{3}$, which are expressed in MSCs and mature osteoblasts, have been shown to promote 
osteoblast differentiation via FAK-ERK1/2 signaling and maintain osteoblast survival via activation of PI3K/Akt signaling (24). In addition, $a_{V} \beta_{3}$ expressed in preOCs and mature osteoclasts has been implicated in the regulation of osteoclast attachment (28). The $\alpha_{V} \beta_{3}$ is also essential for osteoclast-mediated matrix degradation, an effect that occurs through collaboration of c-Fms with the ERK/c-Fos signaling pathway. Hotchkiss et al. suggested that activation of $\alpha_{5} \beta_{1}$ and $\alpha_{V} \beta_{3}$ and downstream signaling pathways by TP and 2DDR affects endothelial cell migration (29), leading us to examine the role of integrins in TPmediated bone remodeling. We found that TP/2DDR enhances osteoclast differentiation by interaction with preOC-expressed $\alpha_{V} \beta_{3}$ and inhibits osteoblast differentiation by interacting with MSC-expressed $\alpha_{5} \beta_{1}$ and $\alpha_{V} \beta_{3}$. The interaction of 2DDR with these integrins activated PI3K/Akt, but not the ERK1/2 signaling pathway, leading to DNMT3A expression. Collectively these results demonstrate that $\alpha_{V} \beta_{3} / a_{5} \beta_{1}$-mediated signaling plays an important role in bone remodeling through the effects of TP on regulation of osteoclastogenesis and osteoblastogenesis.

There are limitations to this study. Bone remodeling is a complex event in which multiple factors, regulating both formation and resorption, collaborate to maintain bone stability. In myeloma, several other factors, including RANKL, DKK1, macrophage inflammatory protein- $1 a$, and stromal cell-derived factor-1, are known to be dysregulated, and it is not clear how TP may interact with these factors. In addition, although our results with TP inhibitors in animal models show promising results, questions remain about their efficacy in human disease. As these models operate in a murine bone marrow microenvironment, which could be somewhat different from human marrow, it is not clear whether TP inhibitors will be effective in the human marrow environment. Despite these concerns, we think it reasonable to extend these studies to humans as TP inhibitors are now U.S. Food and Drug Administration (FDA)-approved for other purposes. In 2015, a TP inhibitor used in this study, Tipiracil hydrochloride, combined with a nucleoside metabolic inhibitor (Trifluridine) was approved by the FDA for treatment of patients with metastatic colorectal cancer (30). Additionally, several DNMT inhibitors are now in clinical trials for cancer treatment: azacitidine is in phase III trials for myelodysplastic syndromes and acute myeloid leukemia (AML), and decitabine is in phase III trials for AML and chronic myelogenous leukemia (31).

The therapeutic effect of these drugs in myeloma or myeloma-induced bone disease remains unknown, but our data in cell lines, patient cells, and rodent models make a compelling case for a role of TP in the genesis of myeloma-induced bone disease and thus encourage repurposing of these inhibitors. An in-depth analysis of the therapeutic efficacy of TP inhibitors in the SCID-hu mouse model—an excellent model for myeloma translational research because it provides a human-like marrow microenvironment-would be a logical next step. This model could facilitate the translation of these inhibitors into human studies of myeloma bone disease. Because TP is often expressed by other malignancies including breast, prostate, and lung, these findings may also have broader implications for the genesis of bone metastasis caused by these and other tumors. 


\section{MATERIALS AND METHODS}

\section{Study design}

We hypothesized that TP induces bone destruction based on an observation of high TP expression in tumor cells $(12,13)$. In a myeloma setting, this study was designed to evaluate the relationship between TP expression and cancer-associated bone lesions. It encompassed three main objectives: to determine the role of TP in myeloma-induced bone lesions; to elucidate the mechanism of TP-induced bone lesions; and to validate the mechanisms in vivo and in vitro using mouse models and patient samples, respectively. In the first objective, we separated all tested primary myeloma cells and human myeloma cell lines into high- and low-TP expressing cells, injected these cells into mice, and assessed osteoclast-mediated bone resorption and osteoblast-mediated bone formation by radiography and bone histomorphometry. We also knocked down or overexpressed $T P$ in myeloma cells to assess whether modulating TP expression affects bone formation/resorption. TRAP and Alizarin red S staining in co-culture of myeloma cells with MSCs or preOCs determined the importance of TP to bone cell differentiation and activity. In the second aim, methylationspecific PCR (MSP) and bisulfite sequencing PCR (BSP) were performed to analyze DNA methylation in the promoters of $R U N X 2$ and osterix in MSCs or in the promoter of IRF8 in preOCs. We also examined DNMT3A, 2DDR expression, and the integrin-PI3K/Akt signaling pathway in MSCs or preOCs. In the third objective, we evaluated the correlations among TP expression, DNMT3A expression, and bone lesions using samples from randomly selected myeloma patients. Additionally, we confirmed the mechanism using the mouse models. Myeloma-bearing mice were randomly selected for the treatment with two TP inhibitors.

All patient samples were obtained from the Myeloma Tissue Bank of The University of Texas MD Anderson Cancer Center. Bone lesions in humans were characterized by a radiologist who was blinded to the severity of clinical bone disease. The number of samples required to achieve a correlation coefficient (r) $\geq 0.7$, a power of $80 \%$, and the level of significance at 5\% was determined to be at least 7 samples. In mouse studies, sample size, the composition of replicates, and an intermediate end point were based on prior knowledge of the mouse models (14). The sample size was initially estimated using power analysis with our prior knowledge on the bone histomorphometric analysis in myeloma-bearing mice. To ensure a power of $80 \%$ to detect the changes in bone volume between the different TP expression groups with 2-sided type I error rate controlled at 0.05 level, we needed 5 mice per group.

The final end point prior to sacrifice was in accordance with the Institutional Animal Care and Use Committee policies and was predefined. All data were included in the analysis and the criteria for interpretation were established prospectively. Experiments were performed three to five times (as indicated in the figure legends). Animal results were verified by repetition over a 3-year period. 


\section{Cell lines and primary cells}

Primary myeloma cells were isolated from the bone marrow aspirates of newly diagnosed myeloma patients using anti-CD138 antibody-coated magnetic beads (Miltenyi Biotec, Inc). The cells were maintained in RPMI 1640 medium with $10 \%$ fetal bovine serum. Normal plasma cells were isolated from the peripheral blood of healthy donors as previously described (18). Myeloma patient MSCs and monocytes were isolated from bone marrow aspirates and cultured as described before (14). This study was approved by the Institutional Review Board of The University of Texas MD Anderson Cancer Center.

\section{DNMT3A activity analysis}

Nuclear extracts were isolated using the EpiQuik Nuclear Extraction Kit (Epigentek) and 3 $\mu \mathrm{l}$ of nuclear extracts from cells was added to each reaction well according to the manufacturer's protocol. DNMT3A activity was measured using the EpiQuik DNA Methyltransferase Activity/Inhibition Assay Kit (Epigentek) as described previously (32).

\section{Measurement of 2DDR levels}

The relative levels of 2DDR in culture medium and mouse serum were measured as described previously (33). Briefly, the samples were degraded in $1.0 \mathrm{M} \mathrm{HCl}$ at $80^{\circ} \mathrm{C}$, and the absorbance at $261 \mathrm{~nm}$ and at $277 \mathrm{~nm}$ was taken. The concentration was determined based on the calibration curve.

\section{Mouse models}

CB.17 SCID mice purchased from Harlan Laboratories were maintained in American Association of Laboratory Animal Care-accredited facilities. The mouse studies were approved by the Institutional Animal Care and Use Committee of The University of Texas MD Anderson Cancer Center. Cultured myeloma cells $\left(5 \times 10^{5}\right.$ cell $/$ mouse $)$ were injected into the femurs of 6- to 8-week-old SCID mice (34). SCID-hu hosts were established as reported previously (14). Human fetal bone chips were implanted subcutaneously into the right flanks of the mice. Primary myeloma cells $\left(1 \times 10^{6}\right.$ cell/mouse $)$ were injected into the implanted human bone chips to establish myeloma. In some experiments, PBS or 7DX $(200 \mu \mathrm{g} / \mathrm{kg})$ or TPI $(300 \mu \mathrm{g} / \mathrm{kg})$ were injected into the peritoneum of mice three times a week for two weeks beginning 3 weeks after myeloma cell injection.

\section{Monitoring tumor burden and bone lesions in mice}

Sera were collected from the mice weekly and tested for myeloma-secreted M-proteins or light chains by ELISA or for 2DDR levels by spectrophotometric analysis. To measure the size of lytic bone lesions, radiographs were scanned with a Faxitron cabinet X-ray system.

\section{Bone histomorphometry}

SCID mouse femurs or human bone chips of SCID-hu mice were fixed in 10\% neutralbuffered formalin and de-calcified, and the bone sections were stained with toluidine blue or TRAP following standard protocols. To assess dynamic histomorphometric indices, mice were given two injections of $20 \mathrm{mg} / \mathrm{kg}$ calcein (Sigma Aldrich) at 6 and 3 days before dissection. The paraffin-fixed femurs were embedded and sectioned. Both analyses were 
done using BIOQUANT OSTEO software (BIOQUANT Image Analysis Co.). Mouse MSCs and monocytes were isolated and cultured as previously described $(35,36)$.

\section{Statistical analysis}

In directly comparing two sets of quantitative data, statistical significance was determined with SPSS software (version 10.0) using unpaired Student $t$-tests. $P$ value $<0.05$ was considered statistically significant. All results were reproduced in at least three independent experiments.

\section{Supplementary Material}

Refer to Web version on PubMed Central for supplementary material.

\section{Acknowledgments}

We thank the staff at MD Anderson's Small Animal Imaging Facility for their assistance with X-ray imaging.

Funding: This work was supported by the National Cancer Institute R01s (CA190863 and CA193362), the National Cancer Institute UTMDACC SPORE in Multiple Myeloma Career Development Award (CDP-060315) and Developmental Research Program (DRP-00013585), the American Cancer Society Research Scholar Grant (127337-RSG-15-069-01-TBG), the UTMDACC IRG-Basic Research, the Leukemia Research Foundation, the American Society of Hematology, and the National Natural Science Foundation of China Grant No. 81470356.

\section{REFERENCES AND NOTES}

1. Rucci N. Molecular biology of bone remodelling. Clinical cases in mineral and bone metabolism: the official journal of the Italian Society of Osteoporosis, Mineral Metabolism, and Skeletal Diseases. 2008; 5:49-56.

2. Karsenty G, Kronenberg HM, Settembre C. Genetic control of bone formation. Annual review of cell and developmental biology. 2009; 25:629-648.

3. Bruzzaniti A, Baron R. Molecular regulation of osteoclast activity. Reviews in endocrine \& metabolic disorders. 2006; 7:123-139. [PubMed: 16951988]

4. Nishikawa K, Iwamoto Y, Kobayashi Y, Katsuoka F, Kawaguchi S, Tsujita T, Nakamura T, Kato S, Yamamoto M, Takayanagi H, Ishii M. DNA methyltransferase 3a regulates osteoclast differentiation by coupling to an S-adenosylmethionine-producing metabolic pathway. Nature medicine. 2015; 21:281-287.

5. Roodman GD. Biology of osteoclast activation in cancer. Journal of clinical oncology: official journal of the American Society of Clinical Oncology. 2001; 19:3562-3571. [PubMed: 11481364]

6. Roodman GD. Pathogenesis of myeloma bone disease. Leukemia. 2009; 23:435-441. [PubMed: 19039321]

7. Terpos E, Confavreux CB, Clezardin P. Bone antiresorptive agents in the treatment of bone metastases associated with solid tumours or multiple myeloma. BoneKEy Rep. 2015; 4

8. Fulciniti M, Tassone P, Hideshima T, Vallet S, Nanjappa P, Ettenberg SA, Shen Z, Patel N, Tai YT, Chauhan D, Mitsiades C, Prabhala R, Raje N, Anderson KC, Stover DR, Munshi NC. Anti-DKK1 $\mathrm{mAb}(\mathrm{BHQ} 880)$ as a potential therapeutic agent for multiple myeloma. Blood. 2009; 114:371-379. [PubMed: 19417213]

9. Toi M, Atiqur Rahman M, Bando H, Chow LW. Thymidine phosphorylase (platelet-derived endothelial-cell growth factor) in cancer biology and treatment. The Lancet Oncology. 2005; 6:158166. [PubMed: 15737832]

10. Fox SB, Moghaddam A, Westwood M, Turley H, Bicknell R, Gatter KC, Harris AL. Plateletderived endothelial cell growth factor/thymidine phosphorylase expression in normal tissues: an immunohistochemical study. The Journal of pathology. 1995; 176:183-190. [PubMed: 7636628] 
11. Bronckaers A, Gago F, Balzarini J, Liekens S. The dual role of thymidine phosphorylase in cancer development and chemotherapy. Medicinal research reviews. 2009; 29:903-953. [PubMed: 19434693]

12. Moghaddam A, Zhang HT, Fan TP, Hu DE, Lees VC, Turley H, Fox SB, Gatter KC, Harris AL, Bicknell R. Thymidine phosphorylase is angiogenic and promotes tumor growth. Proceedings of the National Academy of Sciences of the United States of America. 1995; 92:998-1002. [PubMed: 7532308]

13. Takebayashi Y, Yamada K, Miyadera K, Sumizawa T, Furukawa T, Kinoshita F, Aoki D, Okumura $\mathrm{H}$, Yamada Y, Akiyama S, Aikou T. The activity and expression of thymidine phosphorylase in human solid tumours. European journal of cancer. 1996; 32A:1227-1232. [PubMed: 8758258]

14. Yang J, He J, Wang J, Cao Y, Ling J, Qian J, Lu Y, Li H, Zheng Y, Lan Y, Hong S, Matthews J, Starbuck MW, Navone NM, Orlowski RZ, Lin P, Kwak LW, Yi Q. Constitutive activation of p38 MAPK in tumor cells contributes to osteolytic bone lesions in multiple myeloma. Leukemia. 2012; 26:2114-2123. [PubMed: 22425892]

15. He J, Liu Z, Zheng Y, Qian J, Li H, Lu Y, Xu J, Hong B, Zhang M, Lin P, Cai Z, Orlowski RZ, Kwak LW, Yi Q, Yang J. p38 MAPK in myeloma cells regulates osteoclast and osteoblast activity and induces bone destruction. Cancer research. 2012; 72:6393-6402. [PubMed: 23066034]

16. Chen CC, Chen LC, Liang Y, Tsang NM, Chang YS. Epstein-Barr virus latent membrane protein 1 induces the chemotherapeutic target, thymidine phosphorylase, via NF-kappaB and p38 MAPK pathways. Cellular signalling. 2010; 22:1132-1142. [PubMed: 20214978]

17. Liu CL, Lim YP, Hu ML. Fucoxanthin enhances cisplatin-induced cytotoxicity via NFkappaBmediated pathway and downregulates DNA repair gene expression in human hepatoma HepG2 cells. Marine drugs. 2013; 11:50-66. [PubMed: 23299493]

18. Yaccoby S, Wezeman MJ, Henderson A, Cottler-Fox M, Yi Q, Barlogie B, Epstein J. Cancer and the microenvironment: myeloma-osteoclast interactions as a model. Cancer research. 2004; 64:2016-2023. [PubMed: 15026338]

19. Takayanagi H, Kim S, Koga T, Nishina H, Isshiki M, Yoshida H, Saiura A, Isobe M, Yokochi T, Inoue J, Wagner EF, Mak TW, Kodama T, Taniguchi T. Induction and activation of the transcription factor NFATc1 (NFAT2) integrate RANKL signaling in terminal differentiation of osteoclasts. Developmental cell. 2002; 3:889-901. [PubMed: 12479813]

20. Yaccoby S. Osteoblastogenesis and tumor growth in myeloma. Leukemia \& lymphoma. 2010; 51:213-220. [PubMed: 20038269]

21. Komori T. Regulation of osteoblast differentiation by transcription factors. Journal of cellular biochemistry. 2006; 99:1233-1239. [PubMed: 16795049]

22. Attwood JT, Yung RL, Richardson BC. DNA methylation and the regulation of gene transcription. Cellular and molecular life sciences: CMLS. 2002; 59:241-257. [PubMed: 11915942]

23. Rishi V, Bhattacharya P, Chatterjee R, Rozenberg J, Zhao J, Glass K, Fitzgerald P, Vinson C. CpG methylation of half-CRE sequences creates C/EBPalpha binding sites that activate some tissuespecific genes. Proceedings of the National Academy of Sciences of the United States of America. 2010; 107:20311-20316. [PubMed: 21059933]

24. Marie PJ. Targeting integrins to promote bone formation and repair. Nature reviews Endocrinology. 2013; 9:288-295.

25. Nakamura I, Pilkington MF, Lakkakorpi PT, Lipfert L, Sims SM, Dixon SJ, Rodan GA, Duong LT. Role of alpha(v)beta(3) integrin in osteoclast migration and formation of the sealing zone. Journal of cell science. 1999; 112(Pt 22):3985-3993. [PubMed: 10547359]

26. Aranda P, Agirre X, Ballestar E, Andreu EJ, Roman-Gomez J, Prieto I, Martin-Subero JI, Cigudosa JC, Siebert R, Esteller M, Prosper F. Epigenetic signatures associated with different levels of differentiation potential in human stem cells. PloS one. 2009; 4:e7809. [PubMed: 19915669]

27. Okano M, Xie S, Li E. Cloning and characterization of a family of novel mammalian DNA (cytosine-5) methyltransferases. Nature genetics. 1998; 19:219-220. [PubMed: 9662389]

28. Rodan SB, Rodan GA. Integrin function in osteoclasts. The Journal of endocrinology. 1997; 154(Suppl):S47-56. [PubMed: 9379137] 
29. Hotchkiss KA, Ashton AW, Klein RS, Lenzi ML, Zhu GH, Schwartz EL. Mechanisms by which tumor cells and monocytes expressing the angiogenic factor thymidine phosphorylase mediate human endothelial cell migration. Cancer research. 2003; 63:527-533. [PubMed: 12543812]

30. Kish T, Uppal P. Trifluridine/Tipiracil (Lonsurf) for the Treatment of Metastatic Colorectal Cancer. P \& T: a peer-reviewed journal for formulary management. 2016; 41:314-325. [PubMed: 27162472]

31. Fahy J, Jeltsch A, Arimondo PB. DNA methyltransferase inhibitors in cancer: a chemical and therapeutic patent overview and selected clinical studies. Expert opinion on therapeutic patents. 2012; 22:1427-1442. [PubMed: 23033952]

32. Majid S, Dar AA, Ahmad AE, Hirata H, Kawakami K, Shahryari V, Saini S, Tanaka Y, Dahiya AV, Khatri G, Dahiya R. BTG3 tumor suppressor gene promoter demethylation, histone modification and cell cycle arrest by genistein in renal cancer. Carcinogenesis. 2009; 30:662-670. [PubMed: 19221000]

33. Garrett ER, Blanch J, Seydel JK. Spectrophotometric analysis of ribose and 2-deoxy-D-ribose alone and in mixtures. Journal of pharmaceutical sciences. 1967; 56:1560-1564. [PubMed: 5588705]

34. Wan X, Li ZG, Yingling JM, Yang J, Starbuck MW, Ravoori MK, Kundra V, Vazquez E, Navone NM. Effect of transforming growth factor beta (TGF-beta) receptor I kinase inhibitor on prostate cancer bone growth. Bone. 2012; 50:695-703. [PubMed: 22173053]

35. Soleimani M, Nadri S. A protocol for isolation and culture of mesenchymal stem cells from mouse bone marrow. Nature protocols. 2009; 4:102-106. [PubMed: 19131962]

36. Yang L, Feng D, Bi X, Stone RC, Barnes BJ. Monocytes from Irf5-/- mice have an intrinsic defect in their response to pristane-induced lupus. Journal of immunology. 2012; 189:3741-3750. 
A

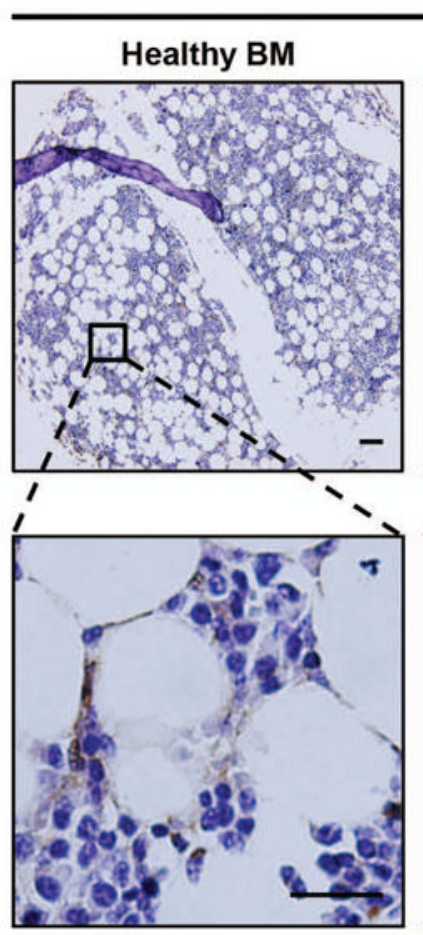

TP

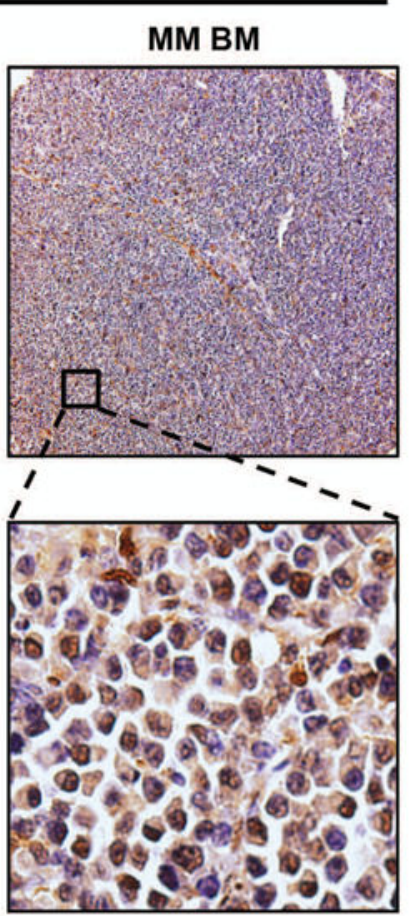

CD138

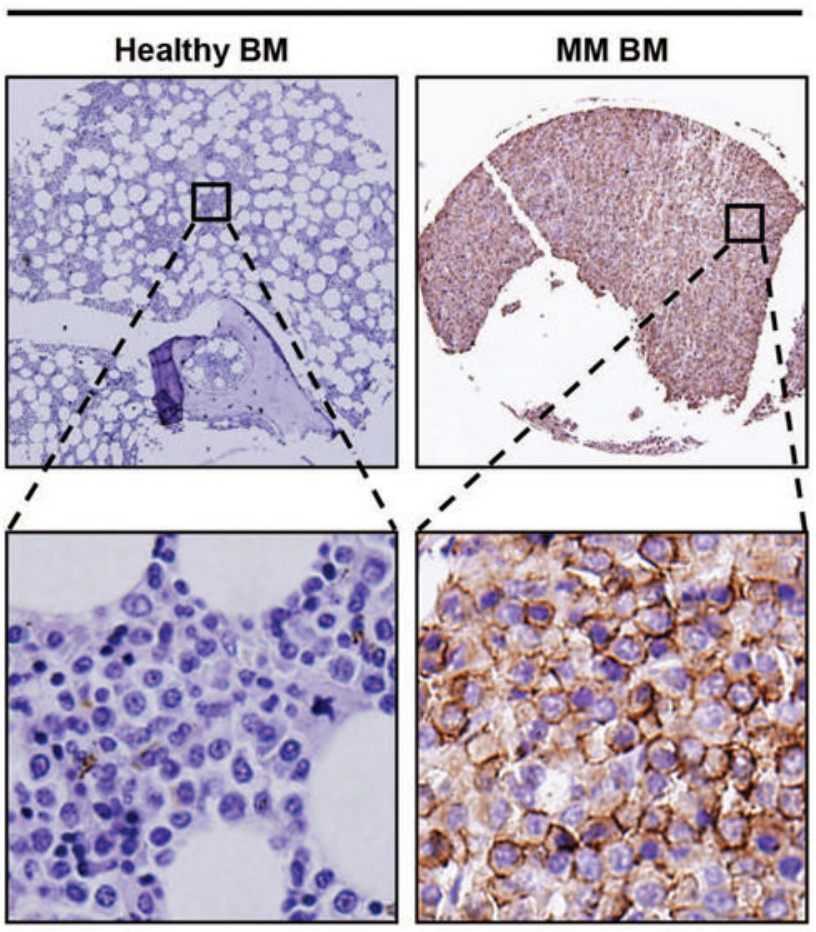

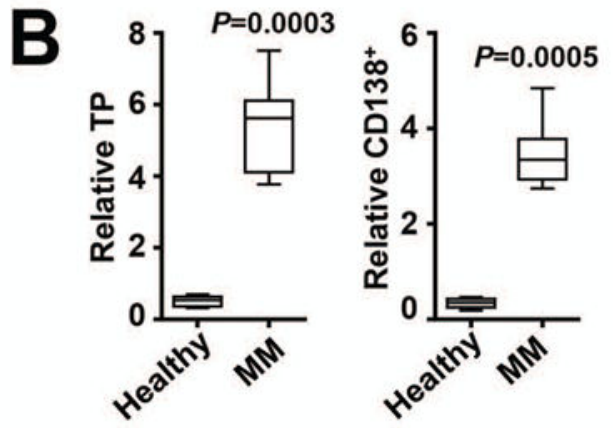

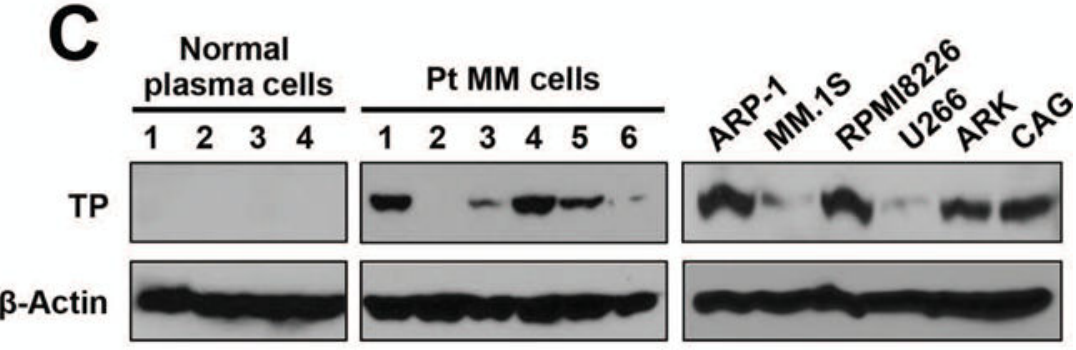

Fig. 1. TP is highly expressed in myeloma

(A) Representative immunohistochemical images of bone marrow biopsies from tissue arrays from 14 healthy and 14 myeloma patients stained for CD138 and TP. (B)

Densitometry analysis of $\mathrm{CD}_{138^{+}}$cells or $\mathrm{TP}^{+}$cells in $(\mathbf{A})$. Data are box plots showing the distribution and median value of quantitative staining $(n=14)$. Scale bars, $50 \mu \mathrm{m}$. $P$ values were determined by Student's $t$ test. (C) Western blot analysis of TP expression in normal plasma cells from 4 healthy donors, malignant plasma cells of 6 myeloma patients, and 6 established human myeloma cell lines. Primary plasma cells were isolated from the bone marrow aspirates of healthy donors or myeloma patients. $\beta$-Actin served as loading control. Data are representative of triplicate blots. 

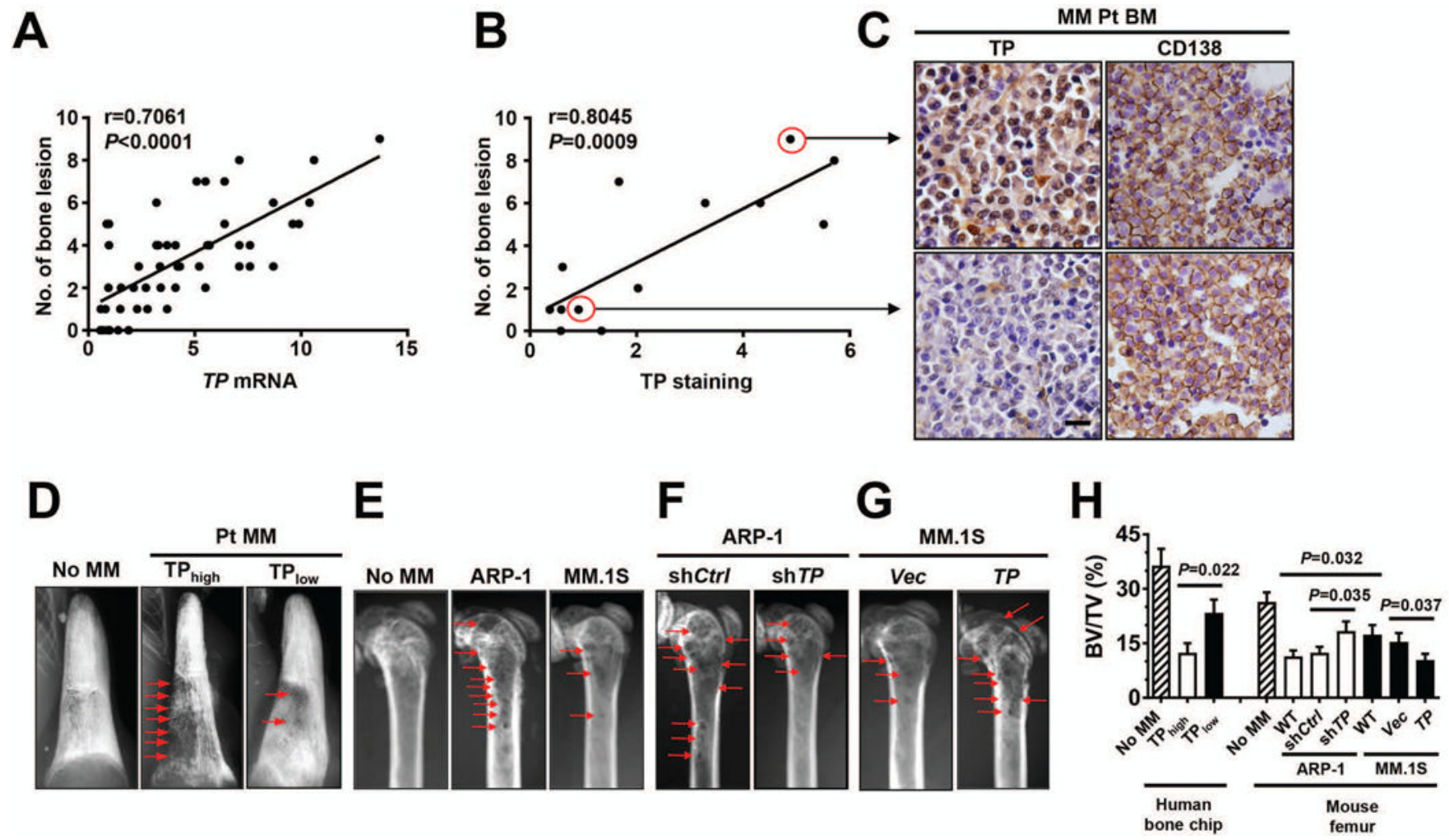

Fig. 2. Association of TP expression and lytic bone lesion in myeloma

(A) Shown is the correlation coefficient between the mRNA levels of $T P$ and numbers of bone lesion in myeloma patients $(n=52)$. $P$ values were determined by Pearson Coefficient. (B and $\mathbf{C}$ ) Bone marrow biopsy samples from $n=13$ patients in (A) were labeled with an anti-TP antibody. TP staining was analyzed using the Image Pro Plus. (B) The correlation between TP staining in bone marrow biopsies and the numbers of bone lesions in myeloma patients. $P$ values were determined by Pearson Coefficient. (C) Representative images of immunohistochemical staining show TP expression in myeloma cells and CD138 ${ }^{+}$infiltrated myeloma cells within bone marrow of the patient samples from (B) highlighted with red circles. Scale bar, $10 \mu \mathrm{m}$. (D to $\mathbf{H}$ ) Based on the levels of TP expression in myeloma cells, patients' myeloma cells were separated into high and low $\mathrm{TP}$ expression groups $\left(\mathrm{TP}_{\text {high }}\right.$ and $\mathrm{TP}_{\text {low; }} n=5$ patients' bone marrow aspirates/group). In addition, myeloma cells were injected into the bone chips of SCID-hu mice or SCID mouse femurs. Shown are representative X-ray images (D-G) and summarized data of the percentage of bone volumes vs total volumes (BV/TV) (H) of lytic lesion in the implanted human bone chips of SCID-hu mice injected with $\mathrm{TP}_{\text {high }}$ and $\mathrm{TP}_{\text {low }}$ cells or in the femurs of SCID mice injected with myeloma cell lines ARP-1 [wild-type (WT), non-targeted shRNA (shCtrl), and TP shRNA $(\operatorname{sh} T P)$ ] and MM.1S [WT, control vector $(V e c)$, and $T P \mathrm{cDNA}(T P)]$. Data are averages $\pm \mathrm{SD}$ ( $n=5$ mice/group, 3 replicate studies). $P$ values were determined by Student's $t$ test. 
A

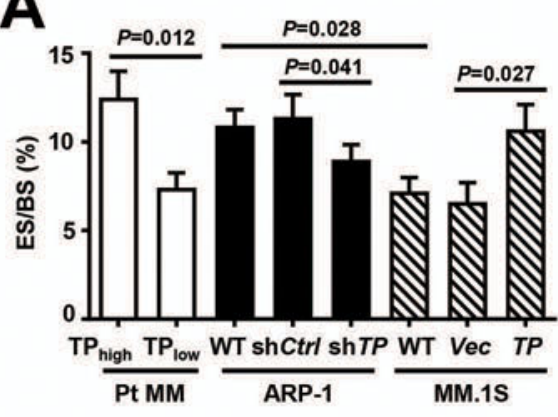

C

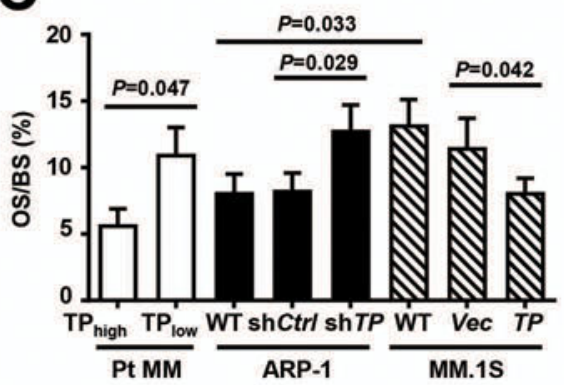

E
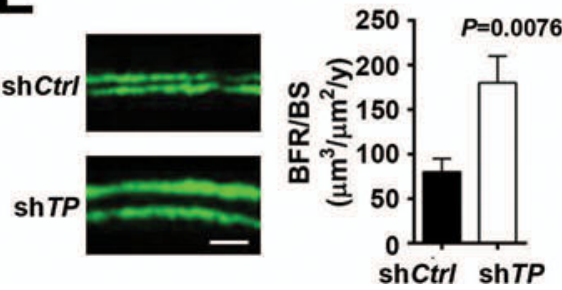

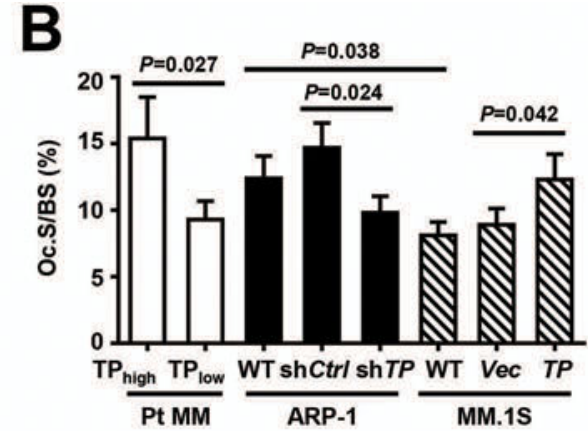

D

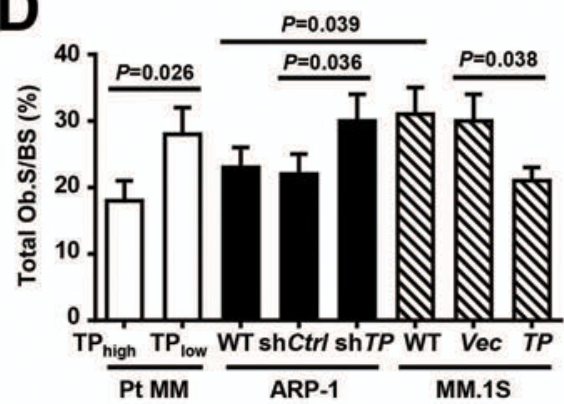

$\mathbf{F}$
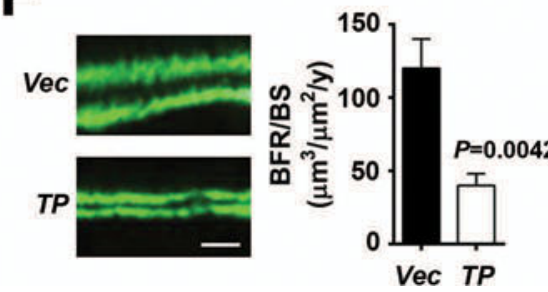

Fig. 3. Myeloma-expressed TP enhances osteoclast-mediated bone resorption and inhibits osteoblast-mediated bone formation in vivo

The implanted human bone chips from SCID-hu mice injected with $\mathrm{TP}_{\text {high }}$ and $\mathrm{TP}_{\text {low }}$ cells ( $n=5$ patients' bone marrow aspirates/group) or the femurs from SCID mice injected with myeloma cell lines ARP-1 (wild-type [WT], non-targeted shRNA [shCtrl], and TP shRNA [sh $T P]$ ) and MM.1S (WT, control vector [ Vec], and TPcDNA [TP]) were fixed, TRAP- or toluidine blue-stained, and analyzed by BIOQUANT OSTEO software. (A to D) The percentage of bone surface eroded by osteoclasts (ES/BS) (A), the percentage of bone surface covered with osteoclasts (Oc.S/BS) (B), the percentage of osteoid surface (OS/BS) (C), and the percentage of total bone surface lined with osteoblasts (Ob.S/BS) (D) in myeloma-bearing human bone chips or mouse femurs. ( $\mathbf{E}$ and $\mathbf{F}$ ) Bone formation rate (BFR/BS) was measured by calcein injection, and the undecalcified bone sections were imaged and analyzed. Shown are representative images or summarized data of bone formation in the femurs from SCID mice injected with myeloma cell lines ARP-1 (sh Ctrl and sh TP) and MM.1S ( Vec and TP). Scale bar, $20 \mu \mathrm{m}$. All data are averages $\pm \mathrm{SD}(n=5$ mice/group, 3 replicate studies). All $P$ values were determined by Student's $t$ test. 

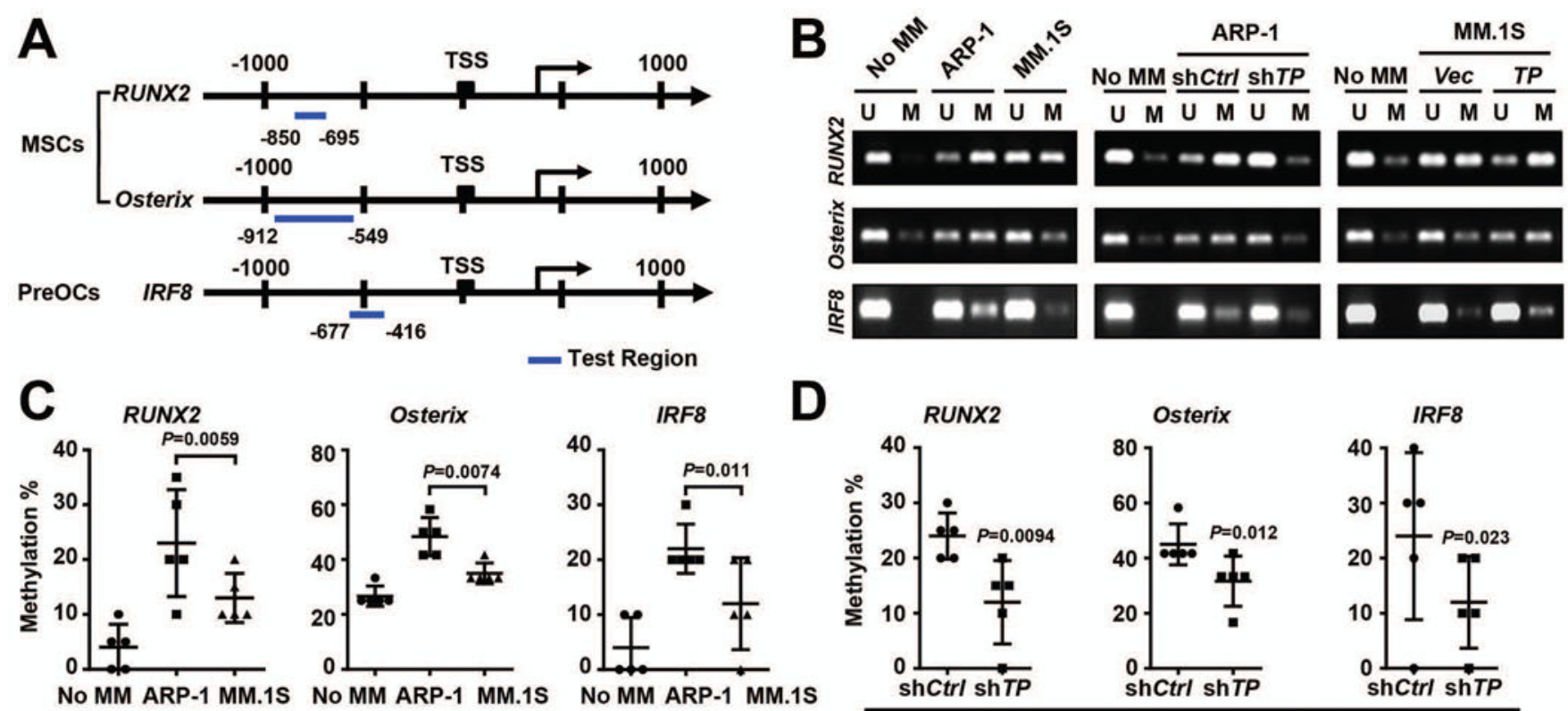

$\mathbf{E}$
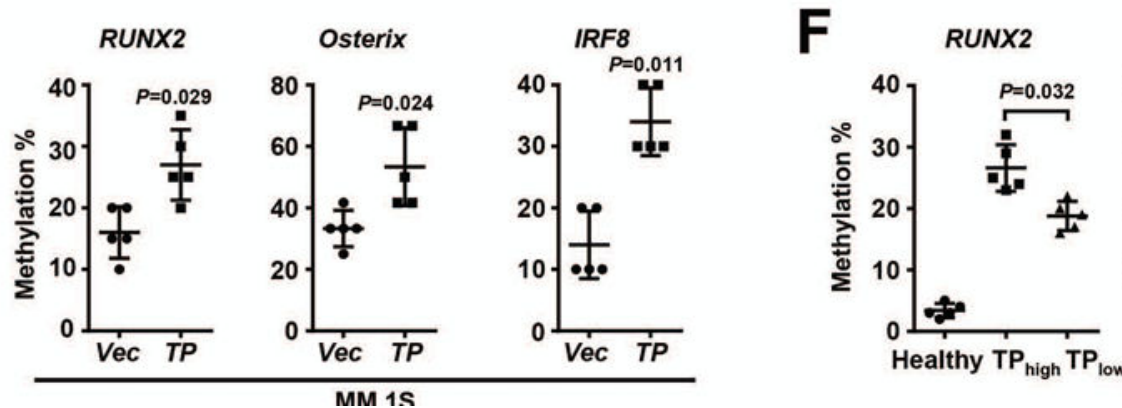

ARP-1

Fig. 4. TP inhibits the expression of RUNX2, osterix, and IRF8 through hypermethylation of their CpG islands

(A) Schematic diagrams of CpG-rich test regions on the promoter of RUNX2 or osterix in human MSCs, and on the promoter of IRF8 in human preOCs. TSS represents the transcription start site. The arrow indicates the translation-initiating ATG site. The CpG-rich test region is marked with a horizontal bar. (B to E) MSCs or preOCs were co-cultured with myeloma cells ARP-1 (wild-type [WT], non-targeted shRNA [sh Ctrl], and TPshRNA [sh TP]) and MM.1S (WT, control vector [ $V e c]$, and TP cDNA [TP]) in their respective medium for 7 days. After cultures, bisulfite-treated genomic DNA was subjected to methylation-specific PCR (MSP) or bisulfite sequencing PCR (BSP) analysis. (B) DNA gel electrophoresis shows the unmethylated (U) and methylated (M) PCR products from MSP analysis. Sequencing results from BSP analysis shows percentage of methylation in the promoter of RUNX2 or osterix in MSCs, and in the promoter of IRF8 in preOCs co-cultured with myeloma cell lines ARP-1 and MM.1S (C), shCtrl or sh TP ARP-1 cells (D), or Vec or TPMM.1S cells (E). Cultured MSCs or preOCs without myeloma (No MM) served as a control. Data are individual samples with averages \pm SD $(n=5)$ of 3 experiments. $P$ values were determined by Student's $t$ test. (F) Summary of BSP analysis shows percentage of methylation in the promoter of RUNX2 or osterix in MSCs, and in the promoter of IRF8 in 
preOCs, of healthy donors and $\mathrm{TP}_{\text {high }}$ or $\mathrm{TP}_{\text {low }}$ patients. Data are individual samples with averages $\pm \mathrm{SD}(n=5)$ of 3 experiments. $P$ values were determined by Student's $t$ test. 


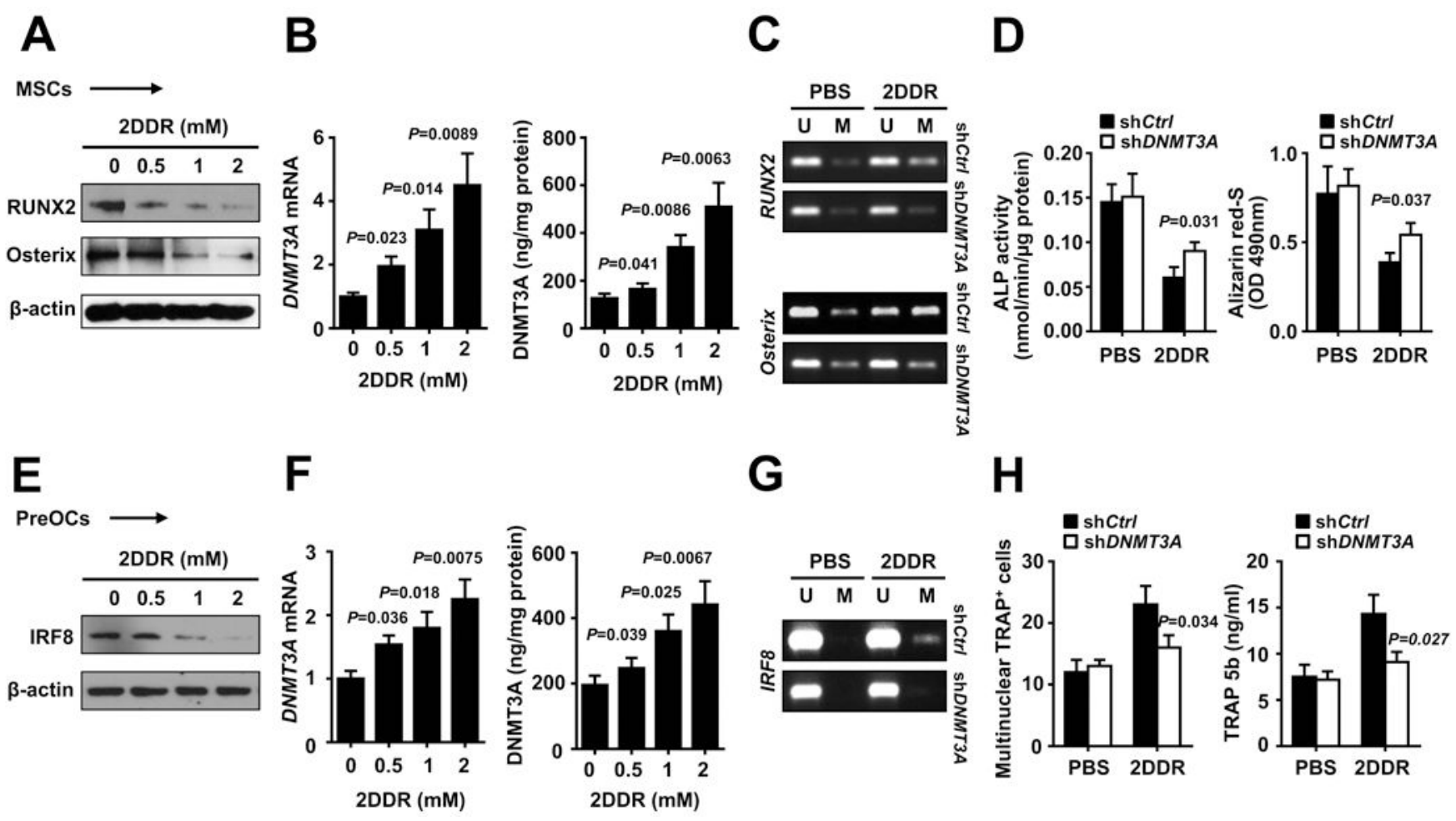

Fig. 5. 2DDR inhibits osteoblast differentiation and activates osteoclast differentiation by upregulating DNMT3A expression

Human MSCs or preOCs were cultured in medium without (0) or with $0.5,1$, or $2 \mathrm{mM}$ of 2DDR for 48 hours. In some studies, MSCs or preOCs carried with non-targeted shRNAs ( $\operatorname{sh} C t r l$ ) or DNMT3A shRNAs ( $\operatorname{sh} D N M T 3 A$ ) were cultured with PBS or $1 \mathrm{mM} 2 \mathrm{DDR}$. (A to D) Expression of RUNX2 and osterix (A), DNMT3A mRNA expression and activity (B), methylation of CGIs in the promoter regions of RUNX2 and osterix (C), and ALP activity and Alizarin red S staining (D) in MSC-derived cells after 2DDR treatment. (E to $\mathbf{H})$ Expression of IRF8 (E), DNMT3A mRNA expression and activity (F), methylation of CGIs in the promoter region of $\operatorname{IRF} 8(\mathbf{G})$, and the number of multiple nuclear ( 3 ) $\mathrm{TRAP}^{+}$cells and secretion of TRAP5b $(\mathbf{H})$ in preOC-derived cells after 2DDR treatment. mRNA expression was normalized to cells without 2DDR (set at 1). The levels of $\beta$-Actin served as loading controls. Data are averages $\pm \mathrm{SD}(n=3)$. $P$ values were determined by Student's $t$ test. Each experiment was repeated three times. 

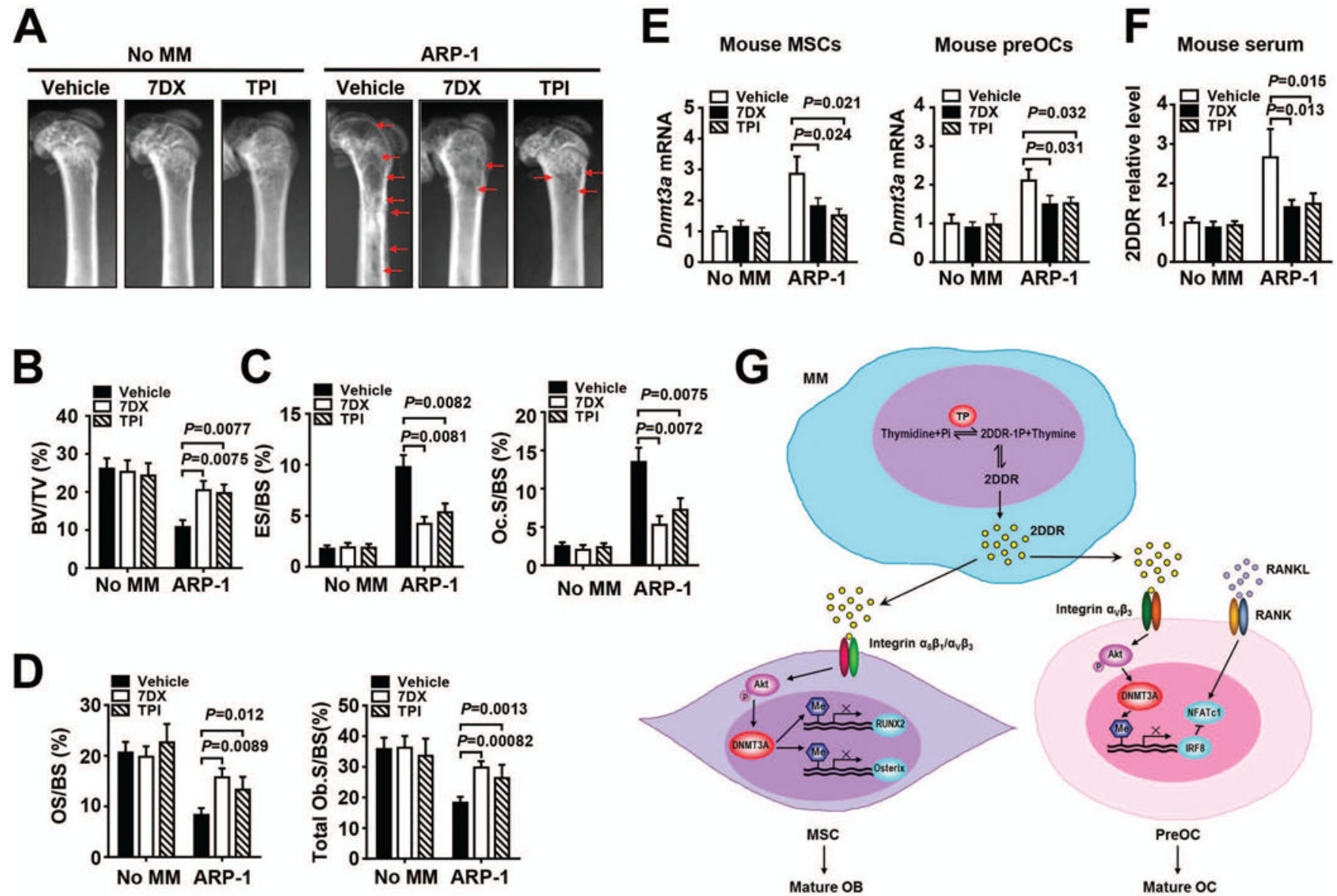

Fig. 6. Administration of TP inhibitor in myeloma-bearing mice reduces bone lesions and osteoclastogenesis and enhances osteoblastogenesis

ARP-1 cells were injected into the femurs of SCID mice. Mice without myeloma cells served as controls (No MM). After 3 weeks, mice were treated with PBS as vehicle controls or TP inhibitor 7DX $(200 \mu \mathrm{g} / \mathrm{kg})$ or TPI $(300 \mu \mathrm{g} / \mathrm{kg})$. After treatment, mice were scanned for radiography, and mouse femurs were subjected to toluidine blue staining or TRAP staining. (A) Representative X-ray images of mouse femurs. (B to D) The percentage of bone volume to total volume (BV/TV) (B), bone surface eroded by osteoclasts (ES/BS) and bone surface covered with osteoclasts (Oc. S/BS) $(\mathbf{C})$, and percentage of osteoid surface (OS/BS) and of bone surface lined with osteoblasts (Ob.S/BS) (D). Data are averages \pm SD ( $n=5$ mice/ group, 3 replicate studies). (E) Dnmt3a mRNA expression in murine MSCs and preOCs isolated from bone marrow aspirates of ARP-1 bearing mice. Data are averages relative to no MM bearing mice (No MM) treated with vehicle (set at 1$) \pm \mathrm{SD}$ ( $n=5$ mice/group, 3 replicate studies). (F) 2DDR levels in the serum of ARP-1 bearing mice. Data are averages relative to that in no $\mathrm{MM}$ bearing mice (No MM) treated with vehicle (set at 1$) \pm \mathrm{SD}(n=5$ mice/group, 3 replicate studies). All $P$ values were determined by Student's $t$ test. (G) A depiction of signaling pathways involved in the myeloma TP-mediated suppression of osteoblastgenesis and activation of osteoclastgenesis. 

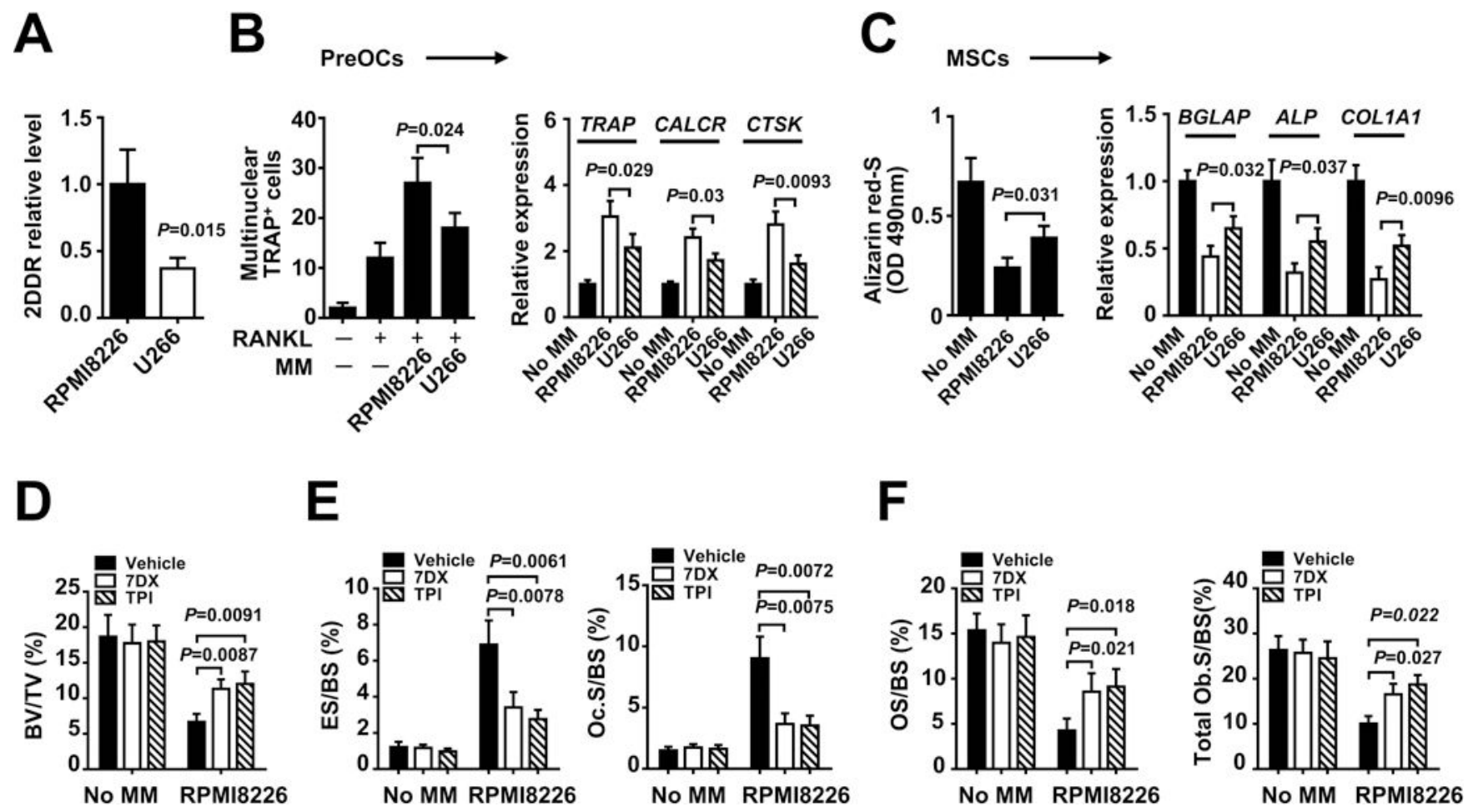

Fig. 7. TP expressed by myeloma cells regulates osteoblast and osteoclast differentiation in vitro and in vivo

(A) Relative levels of 2DDR in human myeloma cell lines RPMI8226 or U266 were measured after 48 hours of culture. Data are relative to RPMI8226. Data are averages \pm SD $(n=3)$ of 3 experiments. (B) PreOCs were cultured alone or co-cultured with RPMI8226 or U266 cells in medium without or with RANKL $(10 \mathrm{ng} / \mathrm{ml})$ for 1 week. preOCs alone without or with $10 \mathrm{ng} / \mathrm{ml}$ of RANKL served as controls. Numbers of multinuclear ( $\geq 3$ ) $\mathrm{TRAP}^{+}$cells and the relative expression of osteoclast differentiation-associated genes $T R A P, C A L C R$, and $C T S K$ were measured. mRNA expression was normalized to cells without myeloma (No MM, set to 1$)$. Data are averages $\pm \operatorname{SD}(n=3)$ of 3 experiments. (C) MSCs were co-cultured with RPMI8226 or U266 in osteoblast medium for 2 weeks and then stained for Alizarin red $\mathrm{S}$. The relative expression of osteoblast differentiation-associated genes $B G L A P, A L P$, and $C O L 1 A 1$ were determined in attached cells. mRNA expression was normalized to cells without myeloma (No MM, set to 1$)$. Data are averages \pm SD $(n=3)$ of 5 experiments. (D to F) RPMI8226 cells $\left(5 \times 10^{5}\right.$ cells/mouse) were injected into the femurs of SCID mice. Mice without myeloma cell injection served as controls (No MM). After 3 weeks, mice were intraperitoneally injected with PBS as vehicle control or the TP inhibitors 7DX $(200 \mu \mathrm{g} / \mathrm{kg})$ or TPI $(300 \mu \mathrm{g} / \mathrm{kg})$ three times per week for 2 weeks. After treatment, mice were scanned for radiography, and mouse femurs were subjected to toluidine blue staining or TRAP staining. We calculated the percentage of bone volume to total volume $(\mathrm{BV} / \mathrm{TV})(\mathrm{D})$, bone surface eroded by osteoclasts $(\mathrm{ES} / \mathrm{BS})$ and bone surface covered with osteoclasts (Oc. S/BS) (E), as well as percentage of osteoid surface (OS/BS) and bone surface lined with osteoblasts (Ob.S/BS) (F). Data are averages \pm SD ( $n=5$ mice/group, 3 replicate studies). All $P$ values were determined by Student's $t$ test. 